\title{
RESEARCH HIGHLIGHT Forks in the road to the first hematopoietic stem cells
}

\author{
Elizabeth D. Howell ${ }^{1,2}$ and Nancy A. Speck (D) \\ Cell Research (2020) 30:457-458; https://doi.org/10.1038/s41422-020-0331-8
}

\begin{abstract}
Hou et al. examine the origin of hemogenic endothelial cells (HECs) by single-cell RNA sequencing. They show that arterial endothelial cells and HECs are derived from a common early arterial endothelial cell precursor, and that some HECs and their immediate progeny retain the capacity to generate both endothelial and hematopoietic cells longer than previously thought.
\end{abstract}

The first hematopoietic stem cells (HSCs) with long-term repopulating potential in mammalian embryos differentiate at mid-gestation from hemogenic endothelial cells (HECs) located primarily in the dorsal aorta through an endothelial-tohematopoietic transition (EHT). ${ }^{1,2}$ The precise molecular identity and source of the endothelial cell (EC) precursors of HECs is not fully understood, although it is widely believed that HECs originate from arterial ECs. ${ }^{3}$

Advances in single cell technologies have allowed for rare heterogenous cell populations to be dissected functionally and molecularly. Live imaging studies of single HECs undergoing EHT showed that they undergo dynamic morphological changes, detach from the endothelium, ${ }^{2}$ and in mammalian embryos accumulate in intra-arterial clusters of hematopoietic cells (IAHCs). Several groups have exploited single-cell qRT-PCR ${ }^{4}$ or single-cell RNA sequencing (scRNA-seq) to identify novel cell surface markers of HECs such as $\mathrm{CD}_{4} 4^{5}$ and to molecularly characterize a transitional cell situated between HEC and HSC called a pre-HSC, which expresses endothelial markers, the cell surface marker c-Kit, and high levels of CD201. ${ }^{6}$

To uncover information about early specification of HECs and the relevant precursors, $\mathrm{Hou}$ et al. utilized scRNA-seq to unbiasedly examine all endothelial populations that spanned continuous stages from E8 to E11 of mouse development. ${ }^{7}$ This time frame encompasses initial dorsal aorta formation, the appearance of HECs, EHT, pre-HSCs and the first HSCs. This strategy allowed for examination of the early cell fate decisions of ECs that give rise to HECs, as well as detailed examination of the HECs themselves. By sampling endothelial populations from several developmental stages, they were able to construct a continual trajectory from primitive ECs to pre-HSCs. The trajectory revealed two major bifurcations along the path from primitive ECs to HECs; primitive ECs must first choose an arterial, but not venous fate, and then early arterial ECs must adopt a hematopoietic fate to become HECs and segregate away from late arterial ECs (Fig. 1a). The identification of an early arterial precursor of both late arterial ECs and HECs argue against the notion that the two lineages arise from distinct precursors. ${ }^{3}$ The arterial endothelial origin of HECs is further supported by data showing that CD44, an arterial endothelial marker, reliably marks HSC-primed HECs in the dorsal aorta from E9.5 to E10.5. ${ }^{5}$ The arterial origin of HSC-primed HECs has also been shown in humans; Zeng et al. showed that this population displayed an unambiguous arterial signature, and IAHCs also expressed arterial genes. ${ }^{8}$ Additionally, NOTCH-mediated arterilization of HECs is required for generating multi-lineage hematopoietic progenitor cells from human pluripotent stem cells. ${ }^{3}$ Taken together, these findings strongly support a model wherein HECs are derived directly from an early arterial endothelial precursor.

Hou et al. also leveraged information from their scRNA-seq data to uncover novel markers of HSC-primed HECs. By comparing different endothelial populations with their putative HEC population, the authors isolated a refined HEC population from E9.5-E10.0 mouse embryos using CD31, CD44, CD201, and c-KIT as markers. This population differentiated into either endothelial tubes or hematopoietic cells in culture, and some single cells produced both (Fig. 1b).

Hou et al. were able to further refine their HEC population by generating a reporter gene from a novel HEC signature gene, Neurl3, that was identified from a bioinformatics screen. They created a Neurl3:EGFP reporter mouse, and observed that EGFP expression was restricted to IAHCs and a subset of aortic ECs, most of which also expressed the transcription factor RUNX1, a known marker of HECs. ${ }^{9}$ Neurl3:EGFP ${ }^{+}$HECs could produce both hematopoietic cells and endothelial tubes in functional assays, and all long-term repopulating HSCs were generated exclusively from Neurl3:EGFP ${ }^{+}$ECs in ex vivo cultures, indicating that Neurl3 is a faithful marker of HSC-primed HECs.

Swiers et al. ${ }^{4}$ previously reported that single HECs isolated from E8.5, E9.5, and E10.5 embryos never gave rise to both endothelial and hematopoietic cells, in contrast to the results reported by Hou et al. ${ }^{7}$. The difference in these results could be due to the markers that the two groups used to isolate HECs; Swiers et al. used a transgenic reporter mouse in which GFP expression is driven from the +23 Runx1 enhancer to isolate Runx1:GFP ${ }^{+}$HECs for their analyses. It is possible that HECs expressing the Runx 1 transgene had lost their dual endothelial-hematopoietic potential, and that Hou et al. have isolated a slightly earlier HEC precursor that is in the midst of hemogenic specification and has not yet extinguished endothelial potential.

The dual-potential HEC described by Hou et al. is distinct from a hemangioblast, a term used to describe an embryonic precursor of both hematopoietic and endothelial cells situated prior to the branch point of arterial and venous ECs. Rather, Hou and colleagues' dual-potential cell appears to be an arterial HEC that is undergoing hemogenic specification, and has not been committed to either the endothelial or hematopoietic cell fates.

\footnotetext{
${ }^{1}$ Department of Cell and Developmental Biology, Abramson Family Cancer Research Institute, Perelman School of Medicine at the University of Pennsylvania, Philadelphia, PA 19104, USA and ${ }^{2}$ Graduate Group in Cell and Molecular Biology, University of Pennsylvania, Philadelphia, PA 19104, USA

Correspondence: Nancy A. Speck (nancyas@upenn.edu)
}

Published online: 12 May 2020 
b

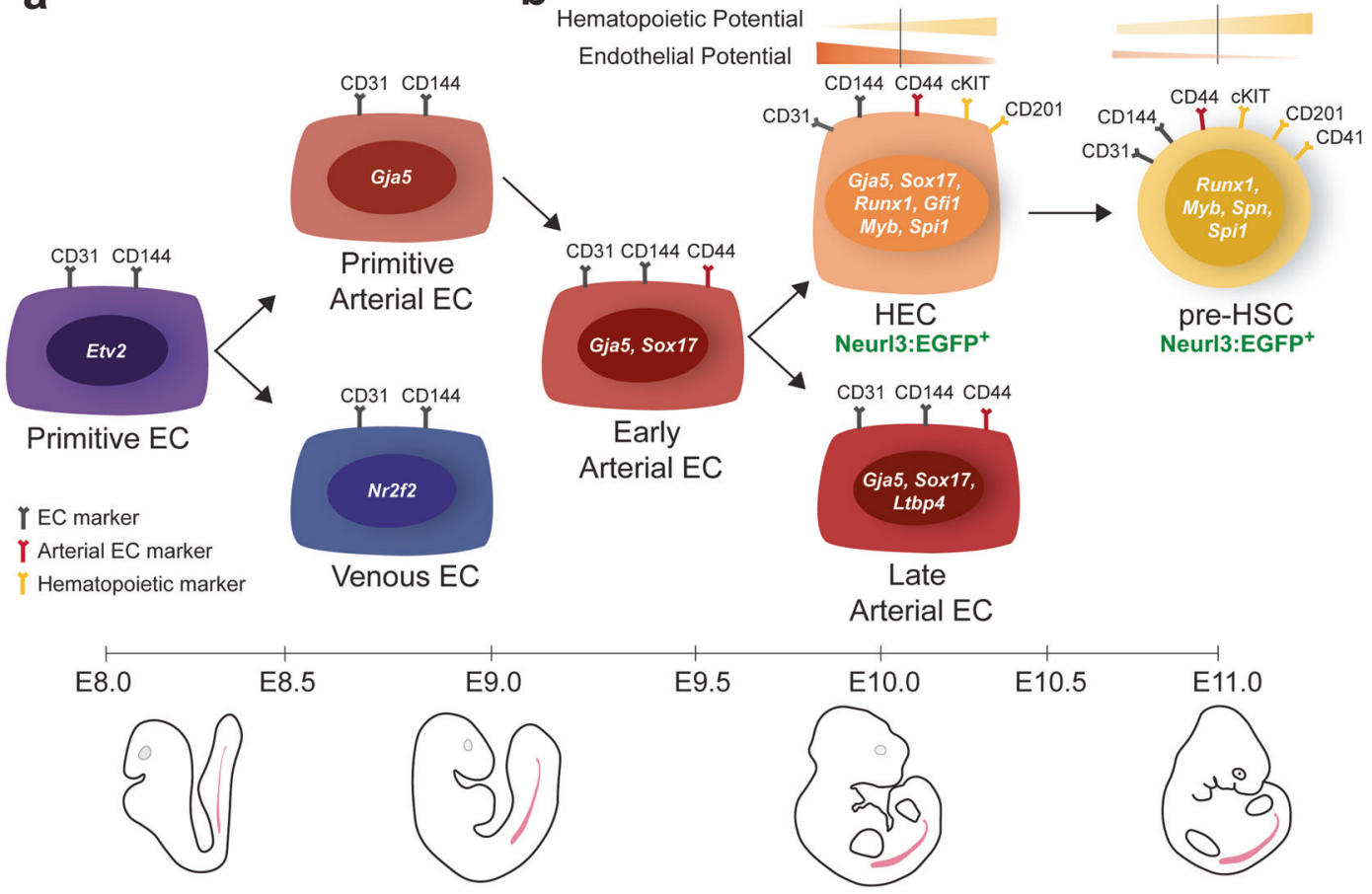

Fig. 1 HSCs and HECs arise from a primitive EC that transits through an arterial EC intermediate and are subsequently marked by the Neurl3:EGFP reporter and possess varying degrees of hematopoietic and endothelial potential. a Primitive ECs (at $\sim$ E8-E8.5) must choose either a arterial or venous fate. Arterial ECs are distinguished by the arterial marker Gja5; venous ECs are distinguished by the venous marker Nr2f2. Primitive arterial ECs continue on to early arterial ECs (between E8.5 and E9.5) that are distinguished by the arterial markers Sox17, Gja5, and surface marker CD44. b Between E9.5 and E10.5 early arterial ECs either adopt a hematopoietic fate and become HECs or maintain their arterial EC fate and become late arterial ECs characterized by arterial markers such as Ltbp4. HECs are characterized by the expression of arterial markers and hematopoietic markers such as Runx1, Gfi1, Myb, and Spi1. The fate of HEC is a pre-HSC ( E10.5-E11.0), which gains cell surface marker CD41 and stronger expression of hematopoietic genes Runx1, Myb, Spn, and Spi1. HECs and pre-HSCs are both marked by the novel HEC signature gene Neurl3, which can be used to isolate these cells using a Neurl3:EGFP reporter mouse. Both HECs and pre-HSCs are functionally characterized by the ability to form both ECs and hematopoietic cells in culture. However, HECs maintain stronger endothelial potential relative to pre-HSCs.

To examine the fate of the HECs cells after EHT, the authors isolated pre-HSCs, the progeny of HSC-producing HECs and a precursor to HSCs. ${ }^{10}$ Strikingly, the authors found that pre-HSCs also contained a low frequency of cells with dual endothelialhematopoietic potential, suggesting that at least a subset of preHSCs are not yet committed to the hematopoietic fate. This is a surprising result, as pre-HSCs are thought to have fully undergone EHT, and extinguished their endothelial fate. An interesting question, then, is at what point in the entire process a cell is finally fully committed to becoming a blood cell?

scRNA-seq has been an invaluable tool for identifying and characterizing rare transient subpopulations that give rise to HSCs. In this study, Hou et al. were able to demonstrate the power of unsupervised bioinformatic analysis from scRNA-seq data to define the immediate precursors of HECs, and to uncover novel markers and regulators of HEC formation.

\section{REFERENCES}

1. Medvinsky, A. \& Dzierzak, E. Cell 86, 897-906 (1996)

2. Kissa, K. \& Herbomel, P. Nature 464, 112-115 (2010).

3. Slukvin, I. I. \& Uenishi, G. I. Exp. Hematol. 71, 3-12 (2019).

4. Swiers, G. et al. Nat. Commun. 4, 2924 (2013).

5. Oatley, M. et al. Nat. Commun. 11, 586 (2020).

6. Zhou, F. et al. Nature 533, 487-492 (2016).

7. Hou, S. et al. Cell Res. https://doi.org/10.1038/s41422-020-0300-2 (2020).

8. Zeng, Y. et al. Cell Res. 29, 881-894 (2019).

9. North, T. et al. Development 126, 2563-2575 (1999).

10. Taoudi, S. \& Medvinsky, A. Proc. Natl. Acad. Sci. USA 104, 9399-9403 (2007). 